\title{
Need of National Indexing System: Challenges and Way Forward
}

\author{
Pawan Kumar Hamal, ${ }^{1}$ Ganesh Dangal, ${ }^{1}$ Arbin Joshi ${ }^{1}$ \\ 'Journal of Nepal Health Research Council, Nepal.
}

\section{BACKGROUND}

Indexing is a replica of quality of journals. We simply understand indexing a journal by an independent authority is a reflection that a particular journal is regular, abides basic norms of publication, free from biases and publishes researches done with good purpose. In Nepal, obligation to have articles published in scientific journal being a criterion for promotion to or acquire any academic positions is one of the single most important factors to propagate scientific publication in Nepal at present. ${ }^{2}$ Reaching with a message to a large number of people could be achieved by publishing the article in quality national journals or specialty journals based on the nature of the research work and the findings ${ }^{3}$ however this idea has been largely overlooked in our local context. Before 2000, as we look at the year of publication in "Nepjol" (Nepal journal online), we had only three medical journals in Nepal, but in next 15 years 29 additional journals were added. So far, exactly half of the journals are published by different specialty societies, associations and research council; but remaining half has been published by medical colleges that mushroomed in last decade in Nepal. There are no journals published by any of the independent publication houses or research bodies, as normally seen in international scenario. "Nepjol" lacks the national consensus and authority of regulation in order to maintain the standards of biomedical journals.

There is a growing need of national indexing body backed up by the national regulation and guidelines which can address the current scenario of unregulated quality research and academic empowerment.

\section{ELEMENTS FOR INDEXING}

\section{Scope of the Journal}

Indexed journals need to be guided by clear aims and visions of scholarly publications. It needs to clearly state the quality of messages and the scientific environment it is trying to foster among its community. A misdirected journal without a clear-cut objective tends to foster predatorship in the publications industry.

\section{Quality of Editorial Policy}

Journal policy should demonstrate that they abide to highest standard of editorial practices in areas of selecting articles, timely and quality peer review process, regularity in publications, adherence to ethical guidelines, disclosures of conflict of interest, timely correspondence, opportunities to audience for commenting and correcting. Journals that do not adhere to editorial integrity and independence in decision making should be discouraged.

\section{Quality of Content of Journal}

Journals prioritizing on original, valid content contributing to the field of science should be considered. The overall content of the journal in various heading along with each article units should be evaluated and follow a proper format and directives. Quality of the layout, printing, graphics, and illustrations should be considered in assessing a journal. Digital object identifier and the concept of open access should be encouraged to improve visibility.

\section{Evaluation of Authorship}

Author responsibility should be guided by ICJME protocol. ${ }^{4}$ Strict regulation and guidelines should be ensured to designate the author and its roles. Journals implementing policy in areas of research misconduct should be encouraged. Predatory journals encouraging authors that pollute the science of publications should be under scanners and punishable.

\section{CHALLENGES}

It is always debated that indexing or inclusion in a particular database as a good surrogate for quality.
DOI:http://dx.doi.org/10.3126/ inhrc.v16i2.20280
Correspondence: Pawan Kumar Hamal, Journal of Nepal Health Research Council, Kathmandu, Nepal. Email: nawaph@gmail.com, Phone: +9779841262246. 
Indexing will not only regulate the quality of the process but will also enhance quality research and improve visibility of its content. However, there are chances that the regulations laid down may not be comfortable to journals of different society, association and colleges which at the moment are enjoying independence and authority. Differences have surfaced over Medical Council of India guidelines in India and were opposed by Indian Association of Medical Editors through a special editorial in Indian Pediatrics. ${ }^{5}$ All journals editors, academic institutions, regulating bodies needs be taken into confidence prior to implementation of the project and made clear about its objectives.

\section{PROPOSED WORKPLAN}

Building a national indexing system is same like building an academic institution which requires expert and trained human resource in field of research, editorials, information technology experts, international stakeholders and association bodies. A stepwise approach to its formation with a clear vision and timeliness should be followed. Early days should be devoted in formation of anauthoritative body endorsed by national act or a board which includes specialist researcher who have worked in this area and understand the whole process of academia. The preliminary report submitted by this team would guide the whole process and bring the issue to surface for discussions and modifications.

Nepal Health Research Council in collaboration with the Ministry of Health along with its international partners and experts can take a lead in this regard. This will help enthusiastic group to guide, train, fund the project during its process of formation and maturation. Multiple discussions with its service partners, academics institutions, in forms of workshops, seminars in part to recognize their challenges, and incorporation in the system can foster new ideas and acceptability among academics.

\section{REFERENCES}

1. Malathi M, Thappa DM. The intricacies of impact factor and mid-term review of editorship. Indian J Dermatol Venereol Leprol. 2012;78:1-4.

2. Magar A. Scientific Publications in Nepal. J Nepal Health Res Counc 2012;10(22):243-9.

3. Dangal G, Hamal PK, Giri M. Understanding Research and Scientific Publication. J Nepal Health Res Counc. 2017;15(1):I-II. DOI: http://dx.doi.org/10.3126/jnhrc.v15i1.18005
4. Defining the Role of Authors and Contributors. http:// www.icmje.org/recommendations/browse/rolesand-responsibilities/defining-the-role-of-authors-andcontributors.html. Accessed 2018 April 05.

5. Aggarwal R, Gogtay N, Kumar R, Sahni P. The Revised Guidelines of the Medical Council of India for Academic Promotions: Need for a Rethink. Indian Pediatrics 2016;53:23-26 